\title{
Mucosal lesions in the human small intestine in shock
}

\author{
U. HAGLUND, L. HULTEN, C. ÅHREN, AND O. LUNDGREN \\ From the Department of Surgery II, Sahlgren's Hospital, the Department of Pathology II and the \\ Department of Physiology, University of Göteborg, Göteborg, Sweden
}

SUMMARY Characteristic mucosal lesions in resected small intestinal segments from seven patients are reported. Preoperatively, four patients were in shock and general hypotension while the three remaining cases showed signs of local intestinal hypotension. The microscopic appearance of the mucosal lesions was in all patients identical with that previously observed in the feline and canine small intestine after haemorrhage or local intestinal hypotension. It is proposed that an extravascular short-circuiting of oxygen in the mucosal countercurrent exchanger and an intravascular aggregation of blood cells might produce tissue hypoxia which makes the mucosa vulnerable to enzymatic degradation.

Haemorrhagic lesions in the small intestinal mucosa have regularly been reported from experimental shock studies on dogs (Wiggers, 1950; Lillehei et al., 1964; Chiu et al., 1970) and cats (Åhrén and Haglund, 1973; Haglund, 1973). A characteristic feature is that these lesions appear first at the tips of the villi, and in cases where the entire villus is destroyed the deeper layers of the intestinal wall are still free from inflammatory changes (Chiu et al., 1970; Åhrén and Haglund, 1973). Similar mucosal lesions have also been reported in patients dying in shock (see, for example, Penner and Bernheim, 1939; Marston, 1962; Bounous, 1969). Most of these studies were, however, 'based on necropsies where postmortem autolysis could not be excluded. There exist in the literature only a few exceptional cases where intestinal mucosal lesions have been reported in patients in shock based on microscopic studies of intestinal segments removed ante-mortem-for example, at operation (Carey et al., 1967; Sørensen and Vetner, 1969; Hugon and Bounous, 1971). This comparatively rare occurrence of reports on intestinal mucosal lesions in association with clinical shock has led to the suggestion that these lesions may be a peculiarity of the dog and, therefore, it has even been proposed that only primates can be used in experimental studies of shock as it appears in clinical medicine (Brobmann et al., 1970; Barton et al., 1972; Swan et al., 1972).

This study reports seven patients exhibiting haemorrhagic lesions in the small intestinal mucosa. The microscopic appearance is described and pos-

Received for publication 24 September 1975. sible pathophysiological mechanisms responsible for the development of such lesions are discussed.

\section{Methods}

\section{CASE HISTORIES}

This report is based on seven patients operated upon for different gastrointestinal disorders. During the preoperative period four patients exhibited obvious signs of clinical shock and, in the remaining three, the small intestine had evidently been subjected to a period of local hypotension. The detailed histories are given below.

Case 1 A 52 year old man had 14 years earlier had a partial gastrectomy and a gastrojejunal anastomosis for bleeding ulcer. The patient was readmitted with clinical signs of gastric bleeding and signs of shock. Blood transfusions could not restore haemoglobin concentration above $8 \mathrm{~g} / \mathrm{dl}$ and for two hours systemic systolic blood pressure remained at $90 \mathrm{~mm}$ $\mathrm{Hg}$. Operation showed a bleeding artery in an ulcer close to the gastrojejunal anastomosis, and this ulcer was excised. On the second postoperative day the patient had a recurrent massive gastric bleeding and developed signs of shock. Despite massive blood transfusions, systolic pressure remained at $70-100$ $\mathrm{mm} \mathrm{Hg}$ for 10 hours, central venous pressure at $0-2$ $\mathrm{cm} \mathrm{H}_{2} \mathrm{O}$, and the pulse rate at $120-140$ beats/min. At the second operation no distinct bleeding could be demonstrated but the mucosa of the jejunum was shown to bleed diffusely and about $150 \mathrm{~cm}$ of the small intestine had to be resected. The resected segment was sent for microscopic investigation. The 
postoperative course was complicated by renal failure and sepsis and the patient died one month later.

Case 2 A 69 year old man had intestinal obstruction and clinical signs of shock. On admission to the hospital, the patient had a systolic blood pressure of $70 \mathrm{~mm} \mathrm{Hg}$, and his pulse rate was 120 beats $/ \mathrm{min}$. In retrospect, the duration of the period of shock was calculated to be at least three hours. Preoperative fluid therapy was initiated during which the status of the patient improved somewhat. At operation the obstruction was dealt with and $50 \mathrm{~cm}$ of the small intestine had to be resected because of adhesions. The resected segment was sent for microscopic examination. The postoperative course was uneventful and the patient left hospital later on in good general condition.

Case 3 A 51 year old woman was admitted to the hospital for ileus caused by a stenosing tumour of the rectum. A decompressing transverse colostomy had to be performed. On the second postoperative day the status of the patient deteriorated markedly with development of peritonitis and shock. Systemic systolic pressure was around $60 \mathrm{~mm} \mathrm{Hg}$, pulse rate 160 beats $/ \mathrm{min}$, and central venous pressure $-1 \mathrm{~cm}$ $\mathrm{H}_{2} \mathrm{O}$. Despite massive fluid therapy, the patient's condition did not improve and a reoperation had to be performed 10 hours later. At laparotomy, a circulatory failure in the colon was revealed and a total colectomy with ileostomy was performed. The small intestine was macroscopically normal but 10 $\mathrm{cm}$ of the terminal part of ileum was available for microscopical investigation. The patient died two weeks after the operation in renal failure and sepsis.

Case 4 A 25 year old man who three years earlier had had a proctocolectomy and ileostomy for Crohn's disease was admitted for recurrent disease in the terminal ileum. A resection was performed and the postoperative course was uneventful. Three weeks later he was readmitted for profuse bleedings through the ileostomy. He was sent via another hospital where blood transfusions were instituted and continued during the ambulance journey. On admission 11 hours after the debut he had clinical signs of shock and treatment with fluid and blood transfusions was intensified. Despite massive fluid therapy, systolic pressure never exceeded $95-100 \mathrm{~mm}$ Hg. At operation four hours later a localized bleeding was demonstrated in the upper jejunum and this intestinal segment was resected and sent for microscopic examination. The postoperative period was uneventful.

Case 5 An 86 year old woman was admitted to the hospital with clinical signs suggesting mesenteric vascular occlusion. At the time of operation the patient had diffuse peritonitis but had not developed general arterial hypotension. The major part of the small intestine was gangrenous and had to be resected, saving about $30 \mathrm{~cm}$ of the proximal jejunum and $60 \mathrm{~cm}$ of the distal ileum which were anastomosed end-to-end. Two to $3 \mathrm{~cm}$ large segments of macroscopically normal intestine at the resection margins were sent for microscopic examination. The patient died eight days later with signs of general circulatory insufficiency.

Case 6 A 59 year old woman with cardiac arrythmia was admitted to the hospital for acute abdominal pains. Peritonitis developed within the first 24 hours but mean arterial blood pressure remained at a normal level. At laparotomy the major part of the small intestine was found to be gangrenous, probably due to an embolus in the superior mesenteric artery. An extensive small bowel resection was performed. A piece of macroscopically normal jejunum at the border to the anastomosis was sent for microscopic investigation. The postoperative course of the patient was complicated and she died four weeks after operation.

Case 7 A 64 year old man was admitted to the hospital with clinical signs of peritonitis and shock. At operation, $70 \mathrm{~cm}$ of the distal ileum was gangrenous and no pulsations in this part of the mesentery were observed. A resection was performed and specimens of the small intestine at the anastomotic borders were sent for microscopical investigation. The patient recovered and left the hospital 10 days later in a good general condition.

\section{HISTOLOGICAL TECHNIQUE}

The resected intestinal segments were immediately mounted on cork and fixed in $10 \%$ neutral formalin. Blocks were cut and prepared for routine paraffin embedment, sliced into $4 \mu \mathrm{m}$ thick sections, mounted and stained according to v. Gieson, with haematoxylin and eosin, and with McManus PAS stain. As described earlier (Chiu et al., 1970; Åhrén and Haglund, 1973), the morphological changes in the mucosa were graded into six grades where grade 0 means normal mucosa and grades $I$ to $\mathrm{V}$ mean increasing damage to the surface epithelium (Table). The pathognomonic lesion for grade I is the development of subepithelial space at the tip of the villus. This space is more extended in grade II where also epithelial lifting is seen. In grade III there is a massive epithelial lifting down the sides of the villus and in grade IV the villus is denuded. Grade V is characterized by disintegration of the lamina propria, 


\begin{tabular}{llll}
\hline Grade & Mucosa & & Submucosa and muscularis \\
\cline { 2 - 4 } & Epithelium & Lamina propria & Normal \\
\hline I & Normal & Normal & Normal \\
II & Subepithelial space at villous tips & Normal & Normal \\
III & Epithelial lifting at villous tips & Normal & Normal \\
IV & Massive epithelial lifting & Occasional haemorrhage & Normal \\
V & Denuded villi & Haemorrhage & Normal \\
\hline
\end{tabular}

Table Microscopic characteristics of grading system used for mucosal lesions

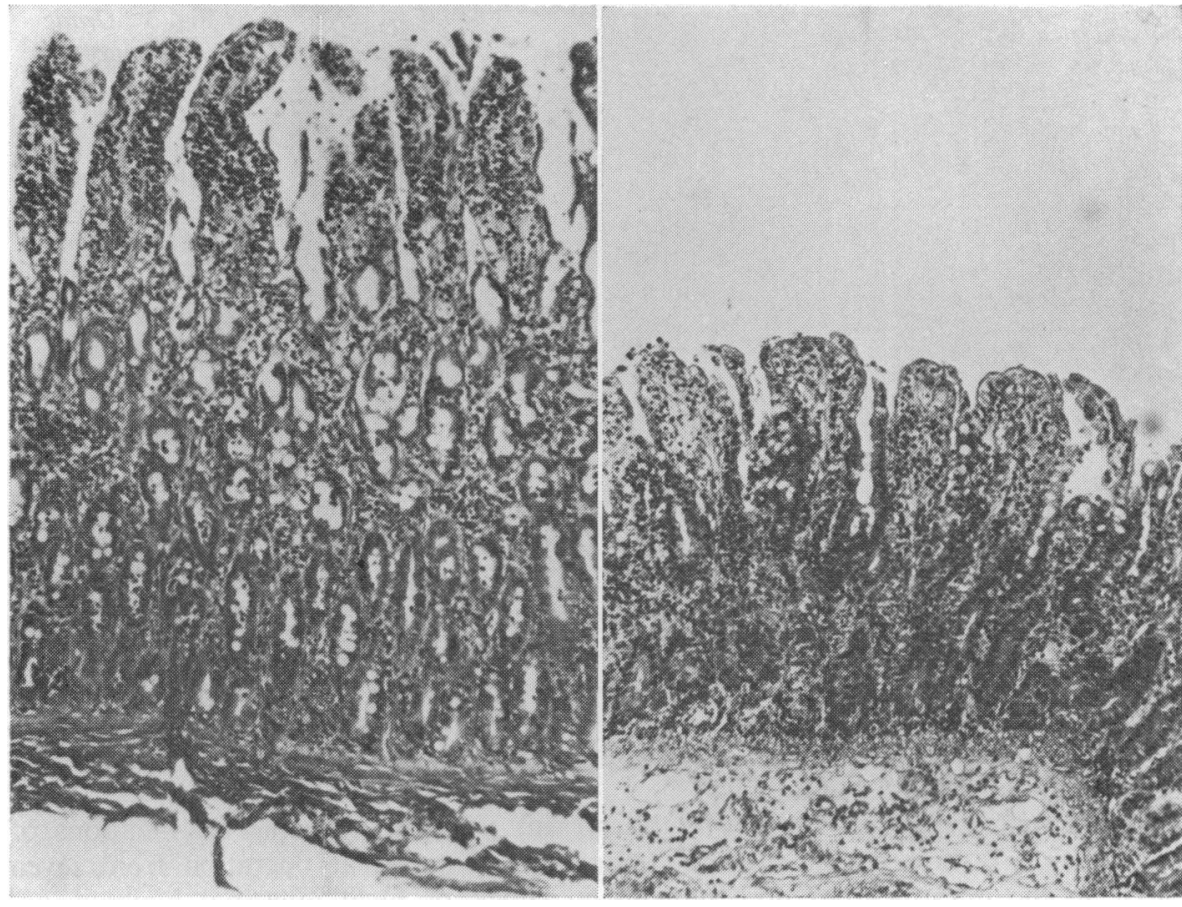

Fig. 1 Grade

II mucosal lesion

(Chiu et al.,

1970) in feline

(left panel) and

human (right

panel) small

intestine.

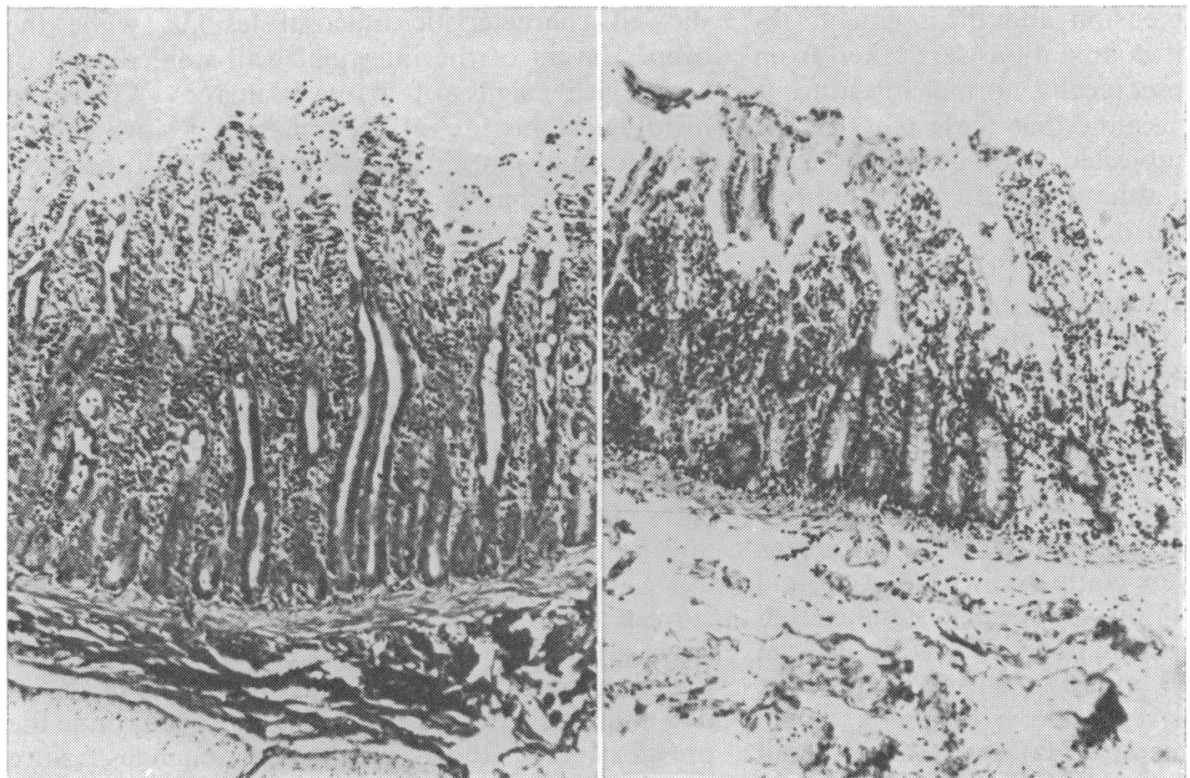

Fig. 2 Grade

III mucosal lesion in feline (left panel) and human (right panel) small intestine. 




Fig. 3 Grade IV-V mucosal lesion in feline (left panel) and human (right panel) small intestine.

haemorrhage, and ulceration in the mucosa but the deeper layers of the intestinal wall are intact (cf. Figs 1, 2, and 3).

\section{Results}

Two types of patients make-up the present study. In one group (cases 1-4) there was no evidence of occlusive vascular disease in the mesenteric vessels but the patients had been hypotensive for varying lengths of time. The other group (cases 5-7) showed signs of occlusive vascular disease in the intestines but no general arterial hypotension and the segments resected for histological examination were taken from the borders of macroscopically normal intestine. It seems reasonable to assume that these parts of the intestine had been subjected to a period of local arterial hypotension, although one cannot entirely exclude a total vascular occlusion.

Both groups of patients exhibited similar lesions at microscopical examination of the intestinal tissue, the mucosal damage varying from grade I to grade V. Typical examples are presented in the right panels of Figs 1, 2, and 3. Even in areas where the villus, was entirely denuded (grade IV to V), the deeper layers of the intestinal wall showed only minimal inflammatory changes.

The microscopical changes in the tissue specimens from these patients were compared with those found in the small intestine of the cat during a standardized simulated shock (local hypotension with arterial inflow pressure at $30 \mathrm{~mm} \mathrm{Hg}$ during continuous stimulation of the regional sympathetic vasoconstrictor nerve fibres at $6 \mathrm{~Hz}$ ) described in detail earlier by Åhrén and Haglund (1973). Identical changes in villous histology were seen in the two species. In both cases, the lesions were strictly confined to the mucosa. This is exemplified in Figs 1, 2, and 3 which show grade II, III, and IV-V lesions in the human and feline small intestine.

\section{Discussion}

In the present study microscopic examinations of gut segments, removed at operation from seven patients in shock or local intestinal hypotension, showed characteristic mucosal lesions, while the deeper layers of the intestinal wall were devoid of histological signs of tissue damage. When the histological picture of the mucosal lesions in the human small intestine was compared with that previously described in experimental studies in the dog (Chiu et al., 1970) and in the cat (Åhrén and Haglund, 1973), they were found to be identical. Closely similar pictures have also been reported from necropsy studies in man (Penner and Bernheim, 1939; Bounous, 1969). Furthermore, Carey et al. (1967) have described one case and Sørensen and Vetner (1969) three cases where intestinal tissue specimens taken at operation from patients in shock showed the same type of microscopic lesions. Hugon and Bounous (1971) have also reported one similar case studied by electron microscopy. Thus, these studies demonstrate that the small intestine in man may, in certain circumstances, exhibit a histological appearance in shock similar to that seen in dog and cat. It is, however, difficult to determine the prevalence of these histological changes, since 
necropsy material cannot be used due to the rapid postmortem autolysis of the small bowel. It should be underlined, however, that grade I-IV lesions may occur without any obvious macroscopic changes of the intestinal mucosa. Hence, the absence of these do not rule out a microscopical damage of the type reported here.

As regards the pathogenesis of these lesions, it has been inferred that they are secondary to an intense nervous vasoconstriction in the mucosa (Lillehei et al., 1967). However, recent studies on the cat suggest that villous blood flow is very little reduced during hypotension (Lundgren and Svanvik, 1973) or during vasoconstrictor fibre stimulation (Svanvik, 1973). Nevertheless, the mucosal lesions observed in the cat seem to be caused by hypoxia since they are prevented by intraluminal perfusion with oxygenated saline during the shock period but not by perfusion with nitrogenated saline (Haglund, Abe, Åhrén, Braide, and Lundgren, to be published). The apparent paradox with hypoxic villous lesions in the face of almost unchanged villous blood flow can be explained by short-circuiting of oxygen in the intestinal countercurrent exchanger at the villous base and in the deeper layers of the mucosa (Lundgren, 1967; Åhrén and Haglund, 1973; Haglund, 1973; Haglund et al., 1973). In the cat, such a 'shunting' has been demonstrated during 'resting' conditions (Kampp et al., 1967). During hypotension plasma mean transit time in the villi is greatly reduced from about $5 \mathrm{~s}$ to about $25 \mathrm{~s}$ (Lundgren and Svanvik, 1973), and the effectiveness of the countercurrent exchanger is correspondingly increased. In this situation, the villous tip is very likely to be anoxic even in the face of unchanged blood supply (Haglund, 1973; Haglund et al., 1973). Blood flow studies on humans have revealed a close qualitative and quantitative similarity between cat and man (Hultén et al., 1972; Hultén et al., 1976) and have also provided experimental support for the existence of an intestinal countercurrent exchanger in the human gut. The latter conclusion is strongly supported by the demonstration of a tissue hyperosmolality in the villous tips in humans (Haljamäe et al., 1973; Jodal and Lundgren, 1975) which suggests the presence of a countercurrent multiplier in the human gut. Hence, it is proposed that the villous lesions reported in this study were, at least in part, caused by an oxygen 'shunting' in the intestinal countercurrent exchanger.

Rheological factors may also contribute to the development of the lesions. It has been claimed by several authors (Brånemark, 1969; Baeckström et al., 1971; Appelgren, 1972) that an intravascular aggregation of red cells and/or platelets may occur, particularly in those parts of a vascular bed where linear flow rate is sluggish. Hence, vascular plugging, when occurring in certain pathological conditions is observed in the small veins where flow rate is slower than in any other part of a vascular bed. As pointed out above, arterial hypotension leads to a markedly reduced linear rate of flow in the vascular loops of the villi enhancing the risk for aggregation of blood cells. In the cat studies referred to above, measures were taken to minimize these rheological disturbances by intermittently administering dextran which has been reported to prevent intravascular plugging (Appelgren, 1972). In five of the cases of this study (cases 1-5) no dextran was given and an intravascular aggregation of blood cells may have contributed to the development of the mucosal lesions.

It has been proposed that the luminal contents are an important factor in the pathophysiology of the intestinal mucosal lesions in shock (Bounous, 1969). According to this hypothesis, the circulatory insufficiency in shock depresses the biosynthesis of mucin (Bounous et al., 1966). When the interphase between the intraluminal contents and the epithelium is changed, the epithelial cells become vulnerable to the trypsin activity of the intestinal juice (Bounous, 1967) and the mucosal lesions are, thus, created. Recent experiments in our laboratory (Haglund, Abe, Braide, Åhrén, and Lundgren: in preparation) also indicate that the intracellular and intraluminal enzymes may be of importance for the development of the pronounced mucosal lesions (grade IV-V), but the key event in the pathogenesis seems to be tissue hypoxia.

This research was sponsored by grants from the Swedish Medical Research Council (B75-4802, 14X-2855, 17X-3117).

\section{References}

Åhrén, C., and Haglund, U. (1973). Mucosal lesions in the small intestine of the cat during low flow. Acta Physiologica Scandinavica, 88, 541-550.

Appelgren, L. (1972). Perfusion and diffusion in shock. Acta Physiologica Scandinavica, Suppl. 378.

Baeckström, P., Folkow, B., Kovách, A. G. B., Löfving, B., and Oberg, B. (1971). Evidence of plugging of the microcirculation following acute haemorrhage. In 6th European Conference on Microcirculation, pp. 16-22. Edited by J. Ditzel, and D. H. Lewis. Karger: Basel.

Barton, R. W., Reynolds, D. G., and Swan, K. G. (1972). Mesenteric circulatory responses to hemorrhagic shock in the baboon. Annals of Surgery, 175, 204-209.

Bounous, G. (1967). Role of the intestinal contents in the pathophysiology of acute intestinal ischemia. American Journal of Surgery, 114, 368-375.

Bounous, G. (1969). 'Tryptic enteritis': its role in the pathogenesis of stress ulcer and shock. Canadian Journal of Surgery, 12, 397-409.

Bounous, G., McArdle, A. H., Hodges, D. M., Hampson, 
L. G., and Gurd, F. N. (1966). Biosynthesis of intestinal mucin in 'shock'. Annals of Surgery, 164, 13-22.

Brånemark, P.-I. (1968). Rheological aspects of low flow states. In Microcirculation as Related to Shock, p. 161. Edited by D. Shepro and G. P. Fulton. Academic Press: New York.

Brobmann, G. F., Ulano, H. B., Hinshaw, L. B., and Jacobson, E. D. (1970). Mesenteric vascular responses to endotoxin in the monkey and dog. American Journal of Physiology, 219, 1464-1467.

Carey, J. S., Okada, F., Monson, D. O., Yao, S. T., and Shoemaker, W. C. (1967). Intestinal infarction in shock with survival after resection. Journal of the American Medical Association, 199, 422-425.

Chiu, C.-J., McArdle, A. H., Brown, R., Scott, H. J., and Gurd, F. N. (1970). Intestinal mucosal lesion in low-flow states. Archives of Surgery, 101, 478-483.

Haglund, U. (1973). The small intestine in hypotension and haemorrhage. An experimental cardiovascular study in the cat. Acta Physiologica Scandinavica, Suppl. 387.

Haglund, U., Lundgren, O., and Svanvik, J. (1973). On the pathogenesis of the intestinal mucosal lesions in shock. Acta Physiologica Scandinavica, 87, 49A-50A.

Haljamäe, H., Jodal, M., and Lundgren, O. (1973). Countercurrent multiplication of sodium in intestinal villi during absorption of sodium chloride. Acta Physiologica Scandinavica, 89, 580-593.

Hugon, J. S., and Bounous, G. (1971). Intestinal lesions in low flow states: electron microscopic study. In Vascular Disorders of the Intestine, pp. 123-144. Edited by S. J. Boley. Appleton and Century Crofts: New York.

Hultén, L., Jodal, M., Lindhagen, J., and Lundgren, $\mathbf{O}$. (1976). Blood flow in the small intestine of cat and man as analyzed by an inert gas wash-out technique. Gastroenterology. (In press).

Hultén, L., Lindhagen, J., and Lundgren, O. (1972). Blood flow in the parallelcoupled vascular sections of the human small intestine analyzed by ${ }^{133} \mathrm{Xe}$ wash-out technique. Microvascular Research, 4, 461. (Abstract).

Jodal, M., and Lundgren, O. (1975). Demonstration of tissue hyperosmolality in the tips of intestinal villi during sodium chloride absorption. Acta Physiologica Scandinavica (In press).

Kampp, M., Lundgren, O., and Nilsson, N. J. (1968). Extravascular shunting of oxygen in the small intestine of the cat. Acta Physiologica Scandinavica, 72, 396-403.

Lillehei, R. C., Dietzman, R. H., and Movsas, S. (1967). The visceral circulation in shock. Gastroenterology, 52, 468-471.

Lillehei, R. C., Longerbeam, J. K., Bloch, J. H., and Manax, W.G. (1964). The nature of irreversible shock: experimental and clinical observations. Annals of Surgery, 160, 682-708.

Lundgren, O. (1967). Studies on blood flow distribution and counter-current exchange in the small intestine. Acta Physiologica Scandinavica, Suppl. 303.

Lundgren, O., and Svanvik, J. (1973). Mucosal hemodynamics in the small intestine of the cat during reduced perfusion pressure. Acta Physiologica Scandinavica, 88, 551-563.

Marston, A. (1962). The bowel in shock. The role of mesenteric arterial disease as a cause of death in the elderly. Lancet, 2, 365-370.

Penner, A., and Bernheim, A. I. (1939). Acute postoperative enterocolitis. A study on the pathologic nature of shock. Archives of Pathology, 27, 966-983.

Sørensen, F. H., and Vetner, M. (1969). Haemorrhagic mucosal necrosis of the gastrointestinal tract without vascular occlusion. Acta Chirurgica Scandinavica, 135, 439448.

Swan, K. G., Barton, R. W., and Reynolds, D. G. (1972). Splanchnic blood flow in experimental shock. In The Fundamental Mechanisms of Shock. Edited by B. G. Cox and L. B. Hinshaw. Plenum: New York.

Svanvik, J. (1973). Mucosal hemodynamics in the small intestine of the cat during regional sympathetic vasoconstrictor activation. Acta Physiologica Scandinavica, 89, 19-29.

Wiggers, C. J. (1950). Physiology of Shock. Commonwealth Fund: New York. 